\title{
Two birds one stone: semaglutide is highly effective against severe psoriasis in a type 2 diabetic patient
}

\section{Gabriele Costanzo1, Salvatore Curatolo ${ }^{2}$, Barbara Busà ${ }^{3}$, Antonino Belfiore ${ }^{1}$ and Damiano Gullo1}

'Endocrine Unit, Department of Clinical and Experimental Medicine, University of Catania, Garibaldi-Nesima Hospital, Catania, Italy, 2Dermatology Unit, Garibaldi Hospital, Catania, Italy, and 3Pharmacy Unit, Garibaldi-Nesima Hospital, Catania, Italy
Correspondence should be addressed to D Gullo

Email

d.gullo@ao-garibaldi.ct.it

\section{Summary}

Semaglutide is a glucagon-like peptide 1 (GLP-1) receptor agonist, approved for the treatment of type 2 diabetes mellitus (T2DM). GLP-1 analogs exert several biological activities connected not only with an insulinotropic effect but also with immunoregulation and reduction of inflammation. A 73-year-old male patient with class III obesity was referred to us for T2DM, which was not controlled with metformin therapy. He had suffered from plaque psoriasis for some years and was treated with topical therapy and adalimumab, without success. The psoriasis area and severity index (PASI) was 33.2 (indicating severe psoriasis), and the dermatology life quality index (DLQI) was 26.0 (indicating an extremely negative effect on the patient's life). Semaglutide (starting with $0.25 \mathrm{mg} /$ week for 4 weeks, increased to $0.50 \mathrm{mg} /$ week for 12 weeks, and then to $1 \mathrm{mg}$ /week) was added to metformin. After 4 months, glycemic parameters had improved, and his body weight decreased. Unexpectedly, skin lesions of plaque psoriasis improved. PASI decreased by $19 \%$ compared with baseline and quality of life, assessed with the DLQI, markedly ameliorated. After 10 months, glycemic and obesity parameters, as well as psoriasis, improved further. HbA1c, BMI, and PASI were reduced by $32,16.3$, and $92 \%$, respectively, compared with the baseline. DLQI declined to 0 , meaning there was no effect of plaque psoriasis on the patient's life.

\section{Learning points:}

- Psoriasis in patients with type 2 diabetes is often resistant to therapy.

- We observed an obese patient with type 2 diabetes mellitus who achieved glycemic control and weight loss with the addition of semaglutide to metformin and had a relevant and long-lasting improvement of plaque psoriasis, which was previously resistant to biologic therapy.

- Therapy with semaglutide may be attempted in eligible patients with difficult to treat plaque psoriasis.

\section{Background}

Plaque psoriasis is a chronic inflammatory, recurring, and highly prevalent skin condition. It has been correlated with insulin resistance and type 2 diabetes mellitus (T2DM) since 1975 (1). The prevalence of T2DM in patients with psoriasis ranges from 4.4 to $54 \%$ and is higher in patients with moderate to severe psoriasis compared to mild disease (2). Moreover, observational data suggest a more aggressive T2DM and higher prevalence and risk of micro- and macro-vascular complications among patients vs those without psoriasis $(2,3)$. Although the exact mechanism 
linking T2DM and psoriasis is still unclear, it is proposed that chronic inflammation may play a central role in both conditions (4).

Limited therapeutic choices are available for the management of psoriasis in T2DM patients $(5,6)$. Nonetheless, various studies have shown a significant improvement of skin plaques by following weight loss (7) and better glycemic control (8).

Incretin-based therapies are commonly used for T2DM because the stimulation of insulin secretion and inhibition of glucagon secretion by these agents occur in a glucose-dependent manner, excluding an intrinsic risk for hypoglycemia (8). Semaglutide is a long-acting glucagon-like peptide 1 (GLP-1) receptor agonist, recently approved for the treatment of T2DM (9). In addition to the insulinotropic activity, GLP-1 analogs exert several additional biologic activities related to immunoregulation and reduction of inflammation (10). Here, we describe a case of an obese patient with T2DM and psoriasis in which treatment with semaglutide for glycemic control resulted in unexpected, relevant, and persistent improvement of the psoriasis skin lesions.

\section{Case presentation}

A 73-year-old male, obese, Caucasian patient was diagnosed with T2DM in 2016 and treated with lifestyle modifications and metformin $1000 \mathrm{mg}$ twice daily. He had a chronic obstructive pulmonary disease and was prescribed with inhaled long-acting beta-adrenoceptor agonists and steroids daily. In addition, he had suffered from severe psoriasis since 2014, for which he had consulted many dermatologists during the past few years. In 2019, he was treated with adalimumab, a recombinant human monoclonal anti-tumor necrosis factor, obtaining little effect and thus discontinued this treatment in November 2019.

\section{Investigation}

The patient came to our observation in January 2020. At our first examination, glycated hemoglobin (HbA1c) was 7.9\%, fasting glucose level was $162 \mathrm{mg} / \mathrm{dL}$, and self-monitoring blood glucose showed a poor control of diabetes mellitus. His height, weight, and BMI were $158 \mathrm{~cm}, 106.7 \mathrm{~kg}$, and $42.7 \mathrm{~kg} / \mathrm{m}^{2}$, respectively.

The dermatology consultant photo-documented the skin lesions. Psoriasis area and severity index (PASI) was 33.2, confirming a severe form of psoriasis (11). Dermatology life quality index (DLQI) was 26.0, indicating an extremely negative effect on the patient's life (12).

\section{Treatment}

We added semaglutide to existing metformin therapy at a starting dose of $0.25 \mathrm{mg}$ per week subcutaneously for the first 4 weeks of therapy, then increased the dose to $0.50 \mathrm{mg}$ per week. The maintenance dose of $1 \mathrm{mg}$ once weekly was reached at 16 weeks and was subsequently maintained.

\section{Outcome and follow-up}

The patient was visited by the dermatologist after 4 months (T4) of treatment with metformin + semaglutide. PASI was $8.0(-76.0 \%)$ (Fig. 1). We also reassessed the treatment of diabetes. HbA1c was $6.4 \%$, fasting glucose level was 124 $\mathrm{mg} / \mathrm{dL}$, and BMI was 40.3. The patient reported a significant amelioration of his quality of life (DLQI $=3$, indicating a slightly negative impact of psoriasis on the patient's life).

At 10 months of treatment (T10), HbA1c was 5.4\%, fasting glucose was $98 \mathrm{mg} / \mathrm{dL}$, BMI was 38.3, PASI was 2.6 $(-92.2 \%)$, and DLQI was 0 (no effect of psoriasis on the patient) (Fig. 1).

\section{Discussion}

In our patient, to control T2DM, the long-acting GLP-1 agonist semaglutide was added to metformin with the dose of $0.25 \mathrm{mg} /$ week for 4 weeks, increased to $0.50 \mathrm{mg} /$ week for a further 12 weeks, and to $1 \mathrm{mg}$ once weekly thereafter.

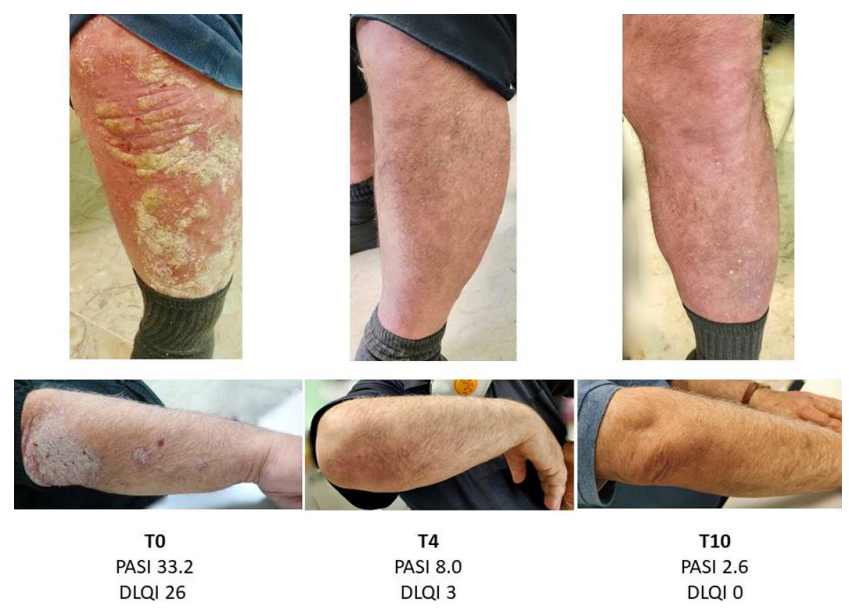

Figure 1

Psoriasis lesions of the upper and lower limbs at baseline (T0) and after 4 (T4) and 10 months (T10) of treatment with semaglutide in addition to metformin. PASI, psoriasis area and severity index; DLQI, dermatology life quality index. 
Semaglutide treatment normalized $\mathrm{HbA1c}$ and induced weight loss, with no significant side effects. During the same period, we observed a rapid improvement of the severe psoriasis lesions at $\mathrm{T} 4$ with a sustained healing response over 10 months. PASI decreased from 32.2 to 8.0 after 4 months and to 2.6 after 10 months (Fig. 1).

Previous to our observation, the patient had been treated for psoriasis with topical medications and adalimumab with unsatisfactory results. Adalimumab had been withdrawn 2 months before we started glucoselowering treatment with semaglutide, due to inefficacy. After realizing the psoriasis improvement achieved after the initiation of semaglutide, the patient refused the reintroduction of adalimumab, reporting his enthusiasm for the 'new' treatment. In fact, the quality of life index, DLQI, dropped to zero, indicating a high level of patient satisfaction with the treatment.

To our knowledge, this is the first report of severe psoriasis in an obese patient with T2DM who has been successfully treated with the use of semaglutide.

GLP-1 analogs have extra-pancreatic effects other than the insulinotropic action; they may suppress cell proliferation, inhibit macrophage migration, impair inflammation by the activation of adenosine 5 '-monophosphate-activated protein kinase $(8,10)$, increase circulating invariant natural killer $\mathrm{T}$ cells, a subset of innate $\mathrm{T}$ cells that exert multiple immunoregulatory functions ( 8 , 10). Finally, GLP-1 analogs may decrease dermal $\gamma \delta$ T-cell number and the expression of IL-17 expression in psoriasis plaques (10). These anti-inflammatory activities may be relevant for the control of diabetes, obesity, and psoriasis lesions, disorders that are known to be correlated with chronic inflammation. A once-daily injection of GLP-1 receptor agonist liraglutide can reduce psoriasis in T2DM patients with an effect independent of weight loss and glycemic control (13). Obesity is an independent risk factor for psoriasis, and weight loss has a positive impact on the severity of this skin disease (7). Semaglutide has recently been reported as an effective treatment of overweight or obesity in a randomized, placebo-controlled clinical trial (14).

Psoriasis treatment in patients with T2DM is often difficult. In these patients, treatment with semaglutide may offer several advantages over conventional treatment for psoriasis, including monoclonal antibodies, due to lower cost and better tolerability. In addition, a single drug could be effective not only on skin disease but also for controlling diabetes and body weight.

More studies are needed to confirm these findings, but on the basis of our experience, we suggest that the GLP-1 agonist semaglutide is considered as a possible treatment for obese patients with T2DM and psoriasis. Due to its peculiar pharmacokinetic characteristics that allow a good efficacy on glycemic and body weight control with a single weekly dose, semaglutide can represent the possibility of killing two (or more) birds with one stone, targeting both diabetes and psoriasis but also obesity.

\section{Declaration of interest}

The authors declare that there is no conflict of interest that could be perceived as prejudicing the impartiality of this case report.

\section{Funding}

This study did not receive any specific grant from any funding agency in the public, commercial, or not-for-profit sector.

\section{Patient consent}

Written informed consent has been obtained from the patient for publication of the submitted article and accompanying images.

\section{Author contribution statement}

G Costanzo, S Curatolo, and D Gullo were directly involved in the management of the patient. D Gullo, G Costanzo, B Busà, and A Belfiore drafted the case report. All of the authors contributed to and approved the final draft of the report.

\section{Acknowledgements}

The writing assistance for the preparation of this manuscript was provided by Polistudium SRL through a Novo Nordisk S.p.A. unconditional grant. Editorial assistance was provided by Laura Brogelli, PhD, and Aashni Shah, BSC (Polistudium SRL, Milan, Italy). The authors are fully responsible for the contents. Novo Nordisk S.p.A. did not influence and was not involved in data collection, interpretation, and analysis.

\section{References}

1 Binazzi M, Calandra P \& Lisi P. Statistical association between psoriasis and diabetes: further results. Archives for Dermatological Research 1975 254 43-48. (https://doi.org/10.1007/BF00561533)

2 Azfar RS, Seminara NM, Shin DB, Troxel AB, Margolis DJ \& Gelfand JM. Increased risk of diabetes mellitus and likelihood of receiving diabetes mellitus treatment in patients with psoriasis. Archives of Dermatology 2012148 995-1000. (https://doi.org/10.1001/archdermatol.2012.1401)

3 Yeung H, Takeshita J, Mehta NN, Kimmel SE, Ogdie A, Margolis DJ, Shin DB, Attor R, Troxel AB \& Gelfand JM. Psoriasis severity and the prevalence of major medical comorbidity: a population-based study. JAMA Dermatology 2013149 1173-1179. (https://doi.org/10.1001/ jamadermatol.2013.5015)

4 Ryan C \& Kirby B. Psoriasis is a systemic disease with multiple cardiovascular and metabolic comorbidities. Dermatologic Clinics 2015 33 41-55. (https://doi.org/10.1016/j.det.2014.09.004)

5 Gisondi P, Cazzaniga S, Chimenti S, Giannetti A, Maccarone M, Picardo M, Girolomoni G, Naldi L \& Psocare Study Group. Metabolic 
abnormalities associated with initiation of systemic treatment for psoriasis: evidence from the Italian Psocare Registry. Journal of the European Academy of Dermatology and Venereology 201327 e30-e41. (https://doi.org/10.1111/j.1468-3083.2012.04450.x)

6 Kirchgessner TG, Uysal KT, Wiesbrock SM, Marino MW \& Hotamisligil GS. Tumor necrosis factor-alpha contributes to obesityrelated hyperleptinemia by regulating leptin release from adipocytes. Journal of Clinical Investigation 1997100 2777-2782. (https://doi. org/10.1172/JCI119824)

7 Jensen P \& Skov L. Psoriasis and obesity. Dermatology 2016232 633-639. (https://doi.org/10.1159/000455840)

8 Gallwitz B. Glucagon-like peptide-1 analogues for type 2 diabetes mellitus: current and emerging agents. Drugs 201171 1675-1688. (https://doi.org/10.2165/11592810-000000000-00000)

9 Goncalves E \& Bell DS. Efficacy of semaglutide versus liraglutide in clinical practice. Diabetes and Metabolism 201946 155-157. (https:// doi.org/10.1016/j.diabet.2019.10.001)

10 Buysschaert M, Baeck M, Preumont V, Marot L, Hendrickx E, Van Belle A \& Dumoutier L. Improvement of psoriasis during glucagonlike peptide- 1 analogue therapy in type 2 diabetes is associated with decreasing dermal $\gamma \delta$ T-cell number: a prospective case-series study. British Journal of Dermatology 2014171 155-161. (https://doi. org/10.1111/bjd.12886)

11 Hongbo Y, Thomas CL, Harrison MA, Salek MS \& Finlay AY. Translating the science of quality of life into practice: what do dermatology life quality index scores mean? Journal of Investigative Dermatology 2005125 659-664. (https://doi.org/10.1111/j.0022-202X.2005.23621.x)

12 Mrowietz U, Kragballe K, Reich K, Spuls P, Griffiths CE, Nast A, Franke J, Antoniou C, Arenberger P, Balieva F, et al. Definition of treatment goals for moderate to severe psoriasis: a European consensus. Archives of Dermatological Research 2011303 1-10. (https://doi.org/10.1007/ s00403-010-1080-1)

13 Chang G, Chen B \& Zhang L. Efficacy of GLP-1rA, liraglutide, in plaque psoriasis treatment with type 2 diabetes: a systematic review and meta-analysis of prospective cohort and before-after studies. Journal of Dermatological Treatment 2021 1-10. (https://doi.org/10.1080 /09546634.2021.1882658)

14 Wilding JPH, Batterham RL, Calanna S, Davies M, Van Gaal LF, Lingvay I, McGowan BM, Rosenstock J, Tran MTD, Wadden TA, et al. Once-weekly semaglutide in adults with overweight or obesity. New England Journal of Medicine 2021384 989. (https://doi.org/10.1056/ NEJMoa2032183)

Received in final form 15 June 2021

Accepted 27 July 2021 